\title{
1 Title: Multivalency enhances the specificity of Fc-cytokine fusions
}

2 Authors: Brian Orcutt-Jahns ${ }^{1}$, Peter C. Emmel ${ }^{1}$, Eli M. Snyder ${ }^{1}$, Cori Posner ${ }^{2}$, Scott M.

Carlson $^{2}$, Aaron S. Meyer ${ }^{1,3,4,5, *}$

\section{$4 \quad$ Affiliations:}

$5 \quad{ }^{1}$ Department of Bioengineering, University of California, Los Angeles, United States of America

$6 \quad{ }^{2}$ Visterra, Inc., Waltham, MA, United States of America

$7 \quad{ }^{3}$ Department of Bioinformatics, University of California, Los Angeles, United States of America

$8{ }^{4}$ Jonsson Comprehensive Cancer Center, University of California, Los Angeles, United States of America

$9{ }^{5}$ Eli and Edythe Broad Center of Regenerative Medicine and Stem Cell Research, University of

10 California, Los Angeles, United States of America

11 *Corresponding author. Email: ameyer@asmlab.org

12 Classification: Research report

13 Keywords: Cytokines, IL-2, IL-15, IL-7, Immunity, Multivalency

\section{Author Emails/Contact Information:}

15 Brian Orcutt-Jahns: orcuttjahnsbrian@gmail.com

16 Peter C. Emmel: petercemmel@gmail.com

17 Eli M. Snyder: emsnyder@g.ucla.edu

18 Cori Posner: Cori.posner@gmail.com

19 Scott M. Carlson: smcarlson@gmail.com

20 Aaron S. Meyer: ameyer@ucla.edu 
Abstract: The common $\gamma$-chain receptor cytokines are promising immune therapies due to their central role in coordinating the proliferation and activity of various immune cell populations. One of these cytokines, interleukin (IL)-2, has potential as a therapy in autoimmunity but is limited in effectiveness by its modest specificity toward regulatory $\mathrm{T}$ cells $\left(\mathrm{T}_{\mathrm{reg}} \mathrm{s}\right)$. Therapeutic ligands are often made dimeric as antibody Fc fusions to confer desirable pharmacokinetic benefits, with under-explored consequences on signaling. Here, we systematically profiled the signaling responses to a panel of wild type and mutein IL-2 molecules in various Fc fusion configurations. We used a tensor-structured dimensionality reduction scheme to decompose the responses of each cell population to each ligand over a range of time points and cytokine concentrations. We found that dimeric muteins are uniquely specific for $T_{\text {reg }} \mathrm{s}$ at intermediate ligand concentrations. We then compared signaling response across all treatments to a simple, two-step multivalent binding model. Our model was able to predict cellular responses with high accuracy. Bivalent $\mathrm{Fc}$ fusions display enhanced specificity and potency for $\mathrm{T}_{\text {regs }}$ through avidity effects toward IL-2R $\alpha$. We then utilize our model to identify the potential benefits conferred by valency engineering as an additional mechanism for cytokines with optimized therapeutic benefits. In total, these findings represent a comprehensive analysis of how ligand properties, and their consequent effects on surface receptor-ligand interactions, translate to selective activation of immune cell populations. It also identifies a new route toward engineering even more selective therapeutic cytokines.

Significance Statement: Signaling in off-target immune cells has hindered the effectiveness of IL-2 as an immunotherapeutic. We show that bivalent IL-2 muteins exhibit more regulatory T cell-selective signaling than monovalent forms. This altered selectivity is explained by altered surface receptor-ligand binding kinetics and can be quantitatively predicted using a multivalent binding model. Finally, our model shows that even more selective IL-2 therapies may be developed by designing cytokines in higher valency formats, revealing valency as an unexplored mechanism for engineering specific IL-2 responses.

\section{Main Text:}

\section{INTRODUCTION}

49 Cytokines that bind to the common $\gamma$-chain $\left(\gamma_{\mathrm{c}}\right)$ receptor, including interleukin (IL)-2, 4, 7, 9, 15 , 50 and 21, are a critical hub in modulating both innate and adaptive immune responses (1). The cytokine family operates through a common theme of binding private receptors for each ligand before engaging the common $\gamma_{c}$ receptor to induce signaling. A prominent phenotypic outcome of $\gamma_{c}$ receptor signaling is lymphoproliferation, and so the cytokines are often observed to be an endogenous or exogenous mechanism for altering the balance of immune cell types. This phenotype is observed most extremely from loss-of-function or reduced activity mutations in $\gamma_{c}$ which subvert T and NK cell maturation (2). Disruptive mutations in private receptors can lead to more selective reductions in cell types such as regulatory $\mathrm{T}$ cells $\left(\mathrm{T}_{\mathrm{regs}} \mathrm{s}\right)$ with IL-2R $\alpha$ or $\mathrm{T}$ cells with IL-7R $\alpha$ (1). Conversely, activating mutations in these receptors, such as IL-7R $\alpha$, promote cancers such as B and T cell leukemias (3).

The importance of these cytokines to immune homeostasis and challenges in altering their signaling toward specific therapeutic goals have inspired a variety of engineered forms. Perhaps 
toward IL-2, and so IL-2R $\alpha$ affinity tunes the relative amount of signaling toward regulatory versus effector populations, while IL-2R $\beta$ modulates the overall signaling potency (4). In most cases, the wild-type cytokine or mutein is fused to an IgG antibody to take advantage of FcRnmediated recycling for extended half-life. The antibody has also been employed in a more active role by binding to IL-2 to influence its availability to each receptor in so-called immunocytokines (5). Fc fusion has taken many forms such as conjugation to the cytokine in an $\mathrm{N}$-terminal or C-terminal orientation, including one or two cytokines per IgG, and including or excluding Fc effector functions (6). Notably, bivalent cytokine fusions have been shown to be more potent in $\mathrm{T}_{\text {reg-targeted engineered therapies, perhaps through pharmacokinetic or other }}$ means (7). The potential design space for these molecules quickly becomes experimentally intractable without consistent design principles.

To address this challenge, here we systematically evaluate the signaling specificity effects of engineered cytokine alterations, including affinity-altering mutations and $\mathrm{Fc}$-fusion formats. We explore three hypotheses for the observed effect of bivalency in Fc-cytokine fusions. We find that the effect of bivalency can be fully explained by altered binding selectivity toward cell based on their abundances. The signaling specificity of all muteins and Fc-formats match well with a multivalent binding model, both between cell types and across cell-to-cell variation within a cell type. We propose that cytokine valency is an unexplored axis for further enhancing selective signaling responses and that many opportunities for using multivalency engineering exist within

83 the $\gamma_{\mathrm{c}}$ cytokine family.

\section{RESULTS}

\section{Multiple potential mechanisms of bivalency effects}

Bivalent $\gamma_{c}$ cytokines have been shown to display altered potency and cell-type-selective signaling profiles, both in vitro and in vivo (8). This alteration has led to both increases and reductions in the mutein's propensity for immune suppression; these variable outcomes may occur due to the differing affinities of the IL-2 mutein employed in the Fc fusions $(7,8)$. The mechanism by which multivalency induces altered signaling compared to monovalent ligands in the $\gamma_{c}$ signaling pathways has not been systematically explored. The possible origins of the differences between monovalent and bivalent cytokine signaling profiles are varied, and each would influence the use of this alteration toward therapeutic ends.

As a first possibility, multivalent $\gamma_{\mathrm{c}}$ ligands may induce signaling through altered surfacereceptor interactions (Fig. 1a). While monovalent ligands can only bind and ultimately transduce signal through a single receptor complex, multivalent ligands can potentially engage two or more complexes simultaneously. This change in signaling activity ultimately leads to an altered functional affinity, referred to as avidity. Among other effects, avidity leads to varied binding depending on the abundance of target receptors (9). In fact, this has been used with IL-2 to deliver cytotoxic cargo more selectively to $\mathrm{T}_{\text {regs }} \mathrm{(}(10)$.

101 A second possible effect of multivalency is assembly of fundamentally distinct signaling complexes (Fig. 1b). Multivalent ligands, by binding multiple receptor complexes, can increase local receptor concentration leading to increases in downstream signal transduction (11). Clustering multiple IL-2R $\beta / \gamma_{\mathrm{c}} / \mathrm{IL}-2$ complexes may also help promote more potent or distinct forms of signaling compared to a single complex achievable with monovalent IL-2. Composition 
of the active receptor complex is critical to the types of signaling responses observed, exemplified by the distinct signaling of "synthekines" that activate combinations of cytokine receptors (12).

109 Finally, multivalent ligands may have distinct responses due to pharmacokinetic (PK) or cell 110 trafficking changes (Fig. 1c). IL-2 is rapidly cleared primarily through receptor-mediated 111 endocytosis after Fc fusion, meaning that PK is closely tied to its activity (13). Nanoparticles and ligands of higher valency are endocytosed through altered mechanisms, ultimately leading to changes in intracellular degradation rates (14). The PK and in vivo half-lives of $\gamma_{c}$ cytokines are also modulated by their valency, with effects, in turn, on PK (8). Changes in PK are especially important to selective expansion of $\mathrm{T}_{\text {regs }}$ as this seems to be promoted most prominently by sustained signaling, over transient boluses of IL-2 (15). Overall, bivalency can have a multitude effects on the use of IL-2 therapeutically, in what experimental systems it will be observed, and how it might be further exploited, depending upon the mechanism of its effects.

\section{Bivalent Fc-cytokine fusions have distinct cell specificity but shared dynamics}

120 To explore the determinants of IL-2 response in a systematic manner, we stimulated peripheral blood mononuclear cells (PBMCs) collected from a single donor with 13 IL-2 muteins with varying receptor affinities in both monomeric and dimeric Fc-fusion formats (Table S1). Stimulated cells were collected at four time points using 12 treatment concentrations. The PBMCs were then stained for canonical cell type markers and pSTAT5, a read-out of signaling response, allowing us to separate response by cell type. Four different cell types $\left(\mathrm{T}_{\text {reg, }}, \mathrm{T}_{\text {helper}}\right.$, $\mathrm{CD} 8+, \mathrm{NK}$ ) were gated and quantified (Fig. S1a-d). $\mathrm{T}_{\text {regs, }}$ and $\mathrm{T}_{\text {helper }} \mathrm{s}$ were further dissected according to their IL-2R $\alpha$ abundances into low, average, and high expression subpopulations by isolating sub-populations using three evenly logarithmically spaced bins (Fig. S1j).

129 Exploring how dynamic responses vary across responding cell types and ligand treatments is challenging due to the multi-dimensional space of potential origins of variations. Restricting ones' view to a single time point, cell type, or ligand concentration provides only a slice of the picture $(6,16)$. To provide a more comprehensive view of response, we organized our profiling experiments into a four-dimensional tensor, wherein the position along each dimension represented the ligand used, concentration, treatment duration, or cell type in the profiling. We then factored this data using non-negative canonical polyadic (CP) decomposition to derive factors summarizing the influence of each dimension (Fig. 2a). Three components explained roughly $90 \%$ of the variance within the dataset (Fig. 2 b).

138 Factorization separated distinct response profiles into separate components, and the effect of each dimension into separate factors. For instance, component 1 almost exclusively represented responses to wild-type cytokines (Fig. 2c), which were the only ligands which were not Fcconjugated, showing a response primarily at high concentrations (Fig. 2d), with broad specificity (Fig. 2e) and a signaling profile that peaks at 30 minutes and then more rapidly decreases (Fig. 2f). An alternative way to interpret the factorization results is to compare profiles within a single dimension. For example, component 1 led to a less sustained profile of signaling response as compared to the other signaling patterns (Fig. 2f).

146 Remarkably, components 2 and 3 cleanly separated ligands conjugated in bivalent or monovalent

147 forms respectively (Fig. 2c). In fact, ligand valency was represented more prominently than 
148 differences in receptor affinity between muteins. Component 2 had uniquely high $\mathrm{T}_{\text {reg }}$ specificity

149 (Fig. 2e) and was most represented at intermediate concentrations (Fig. 2d). Component 2 was

150 also highly correlated with IL-2R $\alpha$ abundance in subsets of $\mathrm{T}_{\text {reg }}$ and $\mathrm{T}_{\text {helper }}$ cells, suggesting that

151 bivalent molecule's specificity for $\mathrm{T}_{\text {regs }}$ is mediated by their higher abundance of IL-2R $\alpha$.

152 Component 3 had a broad cell response (Fig. 2e) and increased monotonically with concentration

153 (Fig. 2d). Despite these strong differences in specificities both components had nearly identical

154 time dynamics (Fig. 2f). In total, these results indicated ligand valency as a critical determinant

155 of IL-2 specificity, and that while Fc fusion subtly affected the specificity of response,

156 monovalent and bivalent ligands had identical dynamics.

\section{Differences among IL-2 responses are explained by a simple multivalent binding model}

158 With the indication that $\mathrm{T}_{\text {reg }}$ specificity is enhanced by multivalency without changes to signaling dynamics, we sought to evaluate how well cell surface binding on its own could predict response. We employed a two-step, equilibrium, multivalent binding model to predict cellular response to IL-2 muteins by assuming that signaling response was proportional to the amount of active receptor-ligand complexes (17). See methods for specifics about how binding predictions were used to predict pSTAT5 response. We fit this model to our signaling profiling experiments and evaluated its concordance with the data. The only non-scaling fitting parameter, $K_{x}^{*}$, had an optimum at $1.2 \times 10^{-11} \# /$ cell, consistent with that seen for other receptor families $(18-20)$. Overall, we observed remarkable consistency between predicted and observed response $\left(\mathrm{R}^{2}=\right.$ 0.85; Fig. 3b). This high accuracy was maintained within subsets of the data including individual cell types and ligands (Fig. 3c-d). To verify our model's predictions were dependent on the valency of the molecule, we again fit the model enforcing that all ligands were monovalent. This significantly reduced model accuracy for many of the bivalent ligands (Fig. 3e), confirming that multivalent binding was critical in modeling signaling response.

172 To ensure that our model was not simply capturing a trend towards higher signaling with increasing concentration, we examined our model's accuracy within specific cytokine concentrations (Fig. 3f). Our model was unable to predict response at the lowest concentrations as these did not stimulate signaling and were dominated by experimental noise but increased in accuracy at concentrations where any signaling response was observed. Finally, we examined how our model's accuracy varied with time by fitting the model to each time point individually. As expected, given that longer treatments likely involve various compensatory signaling such as the degradation or increased transcription of IL-2 receptor subunits $(21,22)$, we most accurately predicted initial responses (30 mins) with a slight decrease in accuracy over longer timescales (Fig. 3g). In total, multivalent cell surface binding showed quantitative agreement with the pattern of cell-type-specific responses to IL-2 muteins, supporting that the specificity enhancement of bivalency is derived from cell surface binding avidity effects.

\section{Multivalency provides a general strategy for further enhanced signaling selectivity}

Given that a simple binding model accurately predicted cell-type-specific responses to IL-2 and that bivalent, Fc-fused IL-2 muteins have favorable specificity properties, we sought to computationally explore to what extent multivalency might be a generally useful strategy. While monovalent ligand binding scales linearly with receptor abundance, multivalent ligands bind nonlinearly depending upon receptor abundance (23). Thus, multivalent ligands should be able to selectively target cells with uniquely high expression of certain $\gamma_{c}$ family receptors. 
191 Valency enhancements are only apparent with coordinated changes in receptor-ligand binding 192 affinities (24). Therefore, we optimized the receptor affinities of simulated ligands while varying 193 valency. We first designed IL-2 muteins of varying valency to obtain optimal $\mathrm{T}_{\text {reg }}$ specificity

194 (Fig. 4a). As expected, ligand valency increased achievable selectivity past what was achievable using the monovalent cytokine format at any receptor affinity. Higher valency required reduced 196 IL-2R $\alpha$ affinities (Fig. 4b).

197 We then explored whether IL-2 muteins lacking IL-2R $\alpha$ binding could selectively target NK cells, based on their uniquely high expression of IL-2R $\beta$, with similar results; IL-2 muteins of higher valency were predicted to be increasingly selective for activation of NK cells, so long as IL-2R $\beta / \gamma_{c}$ affinity was coordinately decreased (Fig. 4c,d). Finally, we sought to explore $\mathrm{T}_{\text {helper- }}$ selective muteins of IL-7, as they express high amounts of IL-7R $\alpha$ (Fig. S1i). We again found that ligands of higher valency should achieve higher degrees of selectivity for these cells, but that the benefits of valency were less than the targeting of $\mathrm{T}_{\text {regs }}$ or NK cells using IL-2 mutants because CD8+ T cells have similar IL-7R $\alpha$ amounts (Fig. 4e). These benefits were again found to be contingent on decreasing affinity of the IL-7 muteins for IL-7R $\alpha$ at higher valency (Fig. 4f). In total, these results show that valency has unexplored potential for designing cytokines with enhanced therapeutic efficacy and reduced toxicity. They also show the potential benefit of the modeling framework described above in guiding therapeutic development.

\section{DISCUSSION}

210 Here, we have systematically explored how ligand properties determine signaling response across 13 ligands including wild-type IL-2, and both monovalent and bivalent Fc fusion IL-2 variants. A tensor-based dimensionality reduction technique identified the patterns of changing response with ligand properties, revealing that multivalent cytokines have unique specificity but identical dynamics (Fig. 2). The mechanism responsible for this unique specificity was then explored using a multivalent binding model, which able to reproduce cell-type-specific responses to IL-2 muteins with high accuracy, indicating that specificity is derived from surface binding avidity effects (Fig. 3). Through this model, we found that cytokines of higher valency offer even greater cell type selectivity given corresponding affinity adjustments, which should translate to therapies of improved potency and reduced toxicity (Fig. 4).

220 The design of cell-type-selective ligands is complicated by the complex signaling processes by which they induce cellular signaling and ultimately response, which leads to a combinatorial explosion of potential ligand design objectives (25). This work serves to demonstrate the importance of the relatively unexplored axis of ligand development represented by the design of multivalent ligands. Multivalent ligands have several documented effects, including altered signal transduction $(11,26)$, binding avidity, and pharmacokinetics (27) or intracellular trafficking (28). While valency has been extensively explored as a means to introduce binding selectivity based on receptor density $(9,29)$, how this effect interacts with the presence of multiple receptor species and cell signaling is surely more complex. The ability of the binding model described in this work to accurately predict immune cell-type-specific response indicates that the cell-type-specific signaling profiles of these cytokines can still be understood as principally arising through receptor avidity effects at the cell surface (Fig. 3).

232 Our work here may be used to guide the design of IL-2 muteins with high selectively for $\mathrm{T}_{\text {regs }}$, an important criterion in the design of IL-2 based treatments for autoimmune diseases $(30,31)$. By 
optimizing the predicted selectivity of high-valency ligands, we showed that multivalency may be exploited to design more effective IL-2 based therapeutics for use in the clinical setting, where IL-2 based therapies have traditionally struggled (Fig. 4a,b) (32). Combined with the superior in vivo half-life conferred by Fc-conjugated IL-2 muteins, these multivalent therapeutics could potentially be used in an out-patient setting and require less frequent dosing (33). Furthermore, the superior selectivity offered by engineered multivalent ligands will likely further increase their in vivo half-lives, due to a reduction in receptor-mediated clearance by off-target populations. We also demonstrated the potential benefit which multivalency may confer in the selective activation of NK cells, which could lead to similarly improved anti-cancer treatments (34). Our approach here may also be applied to engineer selectivity into other signaling pathways characterized by cell type pleiotropy, such as IL-4/IL-13 or TNF systems $(35,36)$.

While our approach does effectively capture cell type specific responses to IL-2 and IL-2 muteins and can be readily applied to other well-studied signaling families, several challenges in its implementation and broad translation to therapeutic development remain. Our model can be used to generate guidelines for the modulation of receptor-ligand interactions in conjunction with valency engineering to design more selective ligands, but also predicts that there are specific receptor affinities required to achieve these benefits (Fig. 4). While a host of methods have been developed and employed to enhance or ablate cytokine affinity for receptor subunits (37), precisely tuning ligand affinities remains a complicated protein engineering challenge. Furthermore, we have not yet experimentally validated whether the advantages conferred by higher valency ligands in vitro translate to in vivo settings. For example, a research group found that while a bivalent IL-2 molecule conjugated to a diphtheria-based toxin showed an enhanced ability to target and eliminate $\mathrm{T}_{\text {regs }} \mathrm{s}$ in in vitro setting (38), its enhanced selectivity failed to be replicated in in vivo trials and did not eliminate $\mathrm{T}_{\text {regs }}$ any more selectively than its monovalent counterpart (39). However, the increased efficacy and reduced toxicity of a bivalent EGFR-based therapy over its monovalent counterpart in an in vivo setting was demonstrated by the same group, suggesting that the specificity conferred by multivalent ligands can indeed transfer to clinical settings (40). This discrepancy between in vivo and in vitro selectivity indicates that further experimental modeling is undoubtedly necessary to explore the in vivo benefits of multivalent IL-2 therapies.

\section{MATERIALS AND METHODS}

\section{Modeling}

\section{Binding model}

267 Model was formulated as described in Tan et al (24). The monomer composition of a ligand complex was represented by a vector $\boldsymbol{\theta}=\left(\theta_{1}, \theta_{2}, \ldots, \theta_{N_{L}}\right)$, where each $\theta_{i}$ was the number of monomer ligand type $i$ on that complex. Let $C_{\boldsymbol{\theta}}$ be the proportion of the $\boldsymbol{\theta}$ complexes in all ligand complexes, and $\Theta$ be the set of all possible $\boldsymbol{\theta}$ 's. We have $\sum_{\boldsymbol{\theta} \in \Theta} C_{\boldsymbol{\theta}}=1$.

271 The binding between a ligand complex and a cell expressing several types of receptors can be

272 represented by a series of $q_{i j}$. The relationship between $q_{i j}$ 's and $\theta_{i}$ is given by $\theta_{i}=q_{i 0}+$ $273 q_{i 1}+\ldots+q_{i N_{R}}$. Let the vector $\mathbf{q}_{i}=\left(q_{i 0}, q_{i 1}, \ldots, q_{i N_{R}}\right)$, and the corresponding $\boldsymbol{\theta}$ of a binding

274 configuration $\mathbf{q}$ be $\boldsymbol{\theta}(\mathbf{q})$. For all $i$ in $\left\{1,2, \ldots, N_{L}\right\}$, we define $\psi_{i j}=R_{\mathrm{eq}, j} K_{a, i j} K_{x}^{*}$ where $j=$ 
$\left\{1,2, \ldots, N_{R}\right\}$ and $\psi_{i 0}=1$. The relative number of complexes bound to a cell with configuration

$\mathbf{q}$ at equilibrium is

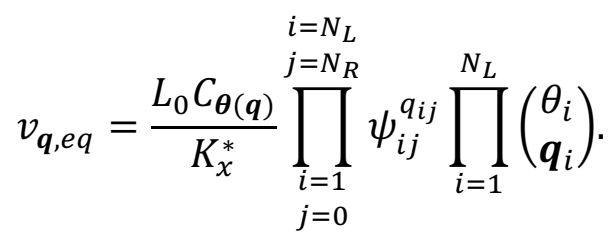

Then we can calculate the relative amount of bound receptor $n$ as

$$
R_{\text {bound }, n}=\frac{L_{0}}{K_{x}^{*}} \sum_{\boldsymbol{\theta} \in \Theta} C_{\boldsymbol{\theta}}\left[\sum_{i=1}^{N_{L}} \frac{\psi_{i n} \theta_{i}}{\sum_{j=0}^{N_{R}} \psi_{i j}}\right] \prod_{i=1}^{N_{L}}\left(\sum_{j=0}^{N_{R}} \psi_{i j}\right)^{\theta_{i}} .
$$

By $R_{\text {tot }, n}=R_{\text {eq }, n}+R_{\text {bound, } n}$, we can solve $R_{\text {eq }, n}$ numerically for each type of receptor.

\section{Application of multivalent binding model to IL-2 signaling pathway}

282

Each IL-2 molecule was allowed to bind to one free IL-2R $\alpha$ and one IL-2R $\beta / \gamma_{c}$ receptor. Initial IL-2-receptor association proceeds with the known kinetics of monomeric ligand-receptor interaction (Table S1). Subsequent ligand-receptor binding interactions then proceed with an association constant proportional to available receptor abundance and affinity multiplied by the scaling constant, $K_{x}^{*}$, as described above. To predict pSTAT5 response to IL-2 stimulation, we assumed that pSTAT5 is proportional to the amount of IL-2-bound IL-2R $\beta / \gamma_{c}$, as complexes which contain these species actively signal through the JAK/STAT pathway. Scaling factors converting from predicted active signaling species to pSTAT5 abundance were fit to experimental data on a per-experiment and cell type basis. A single $K_{x}^{*}$ value was fit for all experiments and cell types.

\section{Tensor Factorization}

Before decomposition, the signaling response data was background subtracted and variance scaled across each cell population. Tensor decomposition was performed using the Python package TensorLy (41), using non-negative canonical polyadic decomposition.

\section{Experimental Methods}

All experimental methods were performed as described in Farhat et al. (42)

\section{Receptor abundance quantitation}

Cryopreserved PBMCs (ATCC, PCS-800-011, lot\#81115172) were thawed to room temperature and slowly diluted with $9 \mathrm{~mL}$ pre-warmed RPMI-1640 medium (Gibco, 11875-093)

supplemented with 10\% fetal bovine serum (FBS, Seradigm, 1500-500, lot\#322B15). Media was removed, and cells washed once more with $10 \mathrm{~mL}$ warm RPMI-1640 + 10\% FBS. Cells were brought to $1.5 \times 10^{6}$ cells $/ \mathrm{mL}$, distributed at 250,000 cells per well in a 96-well V-bottom plate, and allowed to recover $2 \mathrm{hrs}$ at $37^{\circ} \mathrm{C}$ in an incubator at $5 \% \mathrm{CO}$. Cells were then washed twice with PBS + 0.1\% BSA (PBSA, Gibco, 15260-037, Lot\#2000843) and suspended in $50 \mu \mathrm{L}$ PBSA $+10 \%$ FBS for $10 \mathrm{~min}$ on ice to reduce background binding to IgG. 
Antibodies were diluted in PBSA $+10 \% \mathrm{FBS}$ and cells were stained for $1 \mathrm{hr}$ at $4{ }^{\circ} \mathrm{C}$ in darkness with a gating panel (Panel 1, Panel 2, Panel 3, or Panel 4) and one anti-receptor antibody, or an equal concentration of matched isotype/fluorochrome control antibody. Stain for CD25 was included in Panel 1 when CD122, CD132, CD127, or CD215 was being measured (CD25 is used to separate $\mathrm{T}_{\text {regs }}$ from other CD4+ $\mathrm{T}$ cells).

Compensation beads (Simply Cellular Compensation Standard, Bangs Labs, 550, lot\#12970) and quantitation standards (Quantum Simply Cellular anti-Mouse IgG or anti-Rat IgG, Bangs Labs, 815, Lot\#13895, 817, Lot\#13294) were prepared for compensation and standard curve. One well was prepared for each fluorophore with $2 \mu \mathrm{L}$ antibody in $50 \mu \mathrm{L}$ PBSA and the corresponding beads. Bead standards were incubated for $1 \mathrm{hr}$ at room temperature in the dark.

317 Both beads and cells were washed twice with PBSA. Cells were suspended in $120 \mu \mathrm{L}$ per well 318 PBSA, and beads to $50 \mu \mathrm{L}$, and analyzed using an IntelliCyt iQue Screener PLUS with VBR configuration (Sartorius) with a sip time of 35 and 30 secs for cells and beads, respectively. Antibody number was calculated from fluorescence intensity by subtracting isotype control values from matched receptor stains and calibrated using the two lowest binding quantitation standards. $\mathrm{T}_{\text {reg }}$ cells could not be gated in the absence of CD25, so CD4+ T cells were used as the isotype control to measure CD25 in $\mathrm{T}_{\text {reg }}$ populations. Cells were gated as shown in Fig. S1. Measurements were performed using four independent staining procedures over two days. Separately, the analysis was performed with anti-receptor antibodies at $3 \mathrm{x}$ normal concentration to verify that receptor binding was saturated. Replicates were summarized by geometric mean.

\section{pSTAT5 Measurement of IL-2 and -15 Signaling in PBMCs}

Human PBMCs were thawed, distributed across a 96-well plate, and allowed to recover as described above. IL-2 (R\&D Systems, 202-IL-010) or IL-15 (R\&D Systems, 247-ILB-025) were diluted in RPMI-1640 without FBS and added to the indicated concentrations. To measure pSTAT5, media was removed, and cells fixed in $100 \mu \mathrm{L}$ of $10 \%$ formalin (Fisher Scientific, SF100-4) for 15 mins at room temperature. Formalin was removed, cells were placed on ice, and cells were gently suspended in $50 \mu \mathrm{L}$ of cold methanol $\left(-30^{\circ} \mathrm{C}\right)$. Cells were stored overnight at $30^{\circ} \mathrm{C}$. Cells were then washed twice with PBSA, split into two identical plates, and stained $1 \mathrm{hr}$ at room temperature in darkness using antibody panels 4 and 5 with $50 \mu \mathrm{L}$ per well. Cells were suspended in $100 \mu \mathrm{L}$ PBSA per well, and beads to $50 \mu \mathrm{L}$, and analyzed on an IntelliCyt iQue Screener PLUS with VBR configuration (Sartorius) using a sip time of 35 seconds and beads 30 seconds. Compensation was performed as above. Populations were gated as shown in Fig. S1, and the median pSTAT5 level extracted for each population in each well.

\section{Recombinant proteins}

IL-2/Fc fusion proteins were expressed using the Expi293 expression system according to manufacturer instructions (Thermo Scientific). Proteins were as human IgG1 Fc fused at the Nor C-terminus to human IL-2 through a (G4S)4 linker. C-terminal fusions omitted the C-terminal lysine residue of human IgG1. The AviTag sequence GLNDIFEAQKIEWHE was included on whichever terminus did not contain IL-2. Fc mutations to prevent dimerization were introduced into the Fc sequence (43). Proteins were purified using MabSelect resin (GE Healthcare). Proteins were biotinylated using BirA enzyme (BPS Biosciences) according to manufacturer instructions, and extensively buffer-exchanged into phosphate buffered saline (PBS) using 
Amicon $10 \mathrm{kDa}$ spin concentrators (EMD Millipore). The sequence of IL-2R $\beta / \gamma$ Fc heterodimer was based on a reported active heterodimeric molecule (patent application US20150218260A1), with the addition of (G4S)2 linker between the Fc and each receptor ectodomain. The protein was expressed in the Expi293 system and purified on MabSelect resin as above. IL2-R $\alpha$ ectodomain was produced with C-terminal 6xHis tag and purified on Nickel-NTA spin columns (Qiagen) according to manufacturer instructions.

\section{Octet binding assays}

Binding affinity was measured on an Octet RED384 (ForteBio). Briefly, biotinylated monomeric IL-2/Fc fusion proteins were uniformly loaded to Streptavidin biosensors (ForteBio) at roughly $10 \%$ of saturation point and equilibrated for 10 minutes in PBS $+0.1 \%$ bovine serum albumin (BSA). Association time was up to 40 minutes in IL-2R $\beta / \gamma$ titrated in $2 x$ steps from $400 \mathrm{nM}$ to $6.25 \mathrm{nM}$, or IL-2R $\alpha$ from $25 \mathrm{nM}$ to $20 \mathrm{pM}$, followed by dissociation in PBS $+0.1 \%$ BSA. A zero-concentration control sensor was included in each measurement and used as a reference signal. Assays were performed in quadruplicate across two days. Binding to IL-2R $\alpha$ did not fit to a simple binding model so equilibrium binding was used to determine the $\mathrm{K}_{\mathrm{D}}$ within each assay. Binding to IL-2R $\beta / \gamma$ fit a 1:1 binding model so on-rate $\left(\mathrm{k}_{\text {on }}\right)$, off-rate $\left(\mathrm{k}_{\text {off }}\right)$ and $\mathrm{K}_{\mathrm{D}}$ were determined by fitting to the entire binding curve. Kinetic parameters and $\mathrm{K}_{\mathrm{D}}$ were calculated for each assay by averaging all concentrations with detectable binding signal (typically $12.5 \mathrm{nM}$ and above).

\section{Statistical analysis}

The number of replicates performed for each experimental measurement, and the values of confidence intervals are described in corresponding figure captions. $\mathrm{N}$ is used to describe the number of times a particular experiment was performed. All flow cytometry experiments performed on hPBMCs were conducted using separate experimental replicates on cells gathered from a single donor. To quantify population-level flow cytometry measurements for both signaling and receptor quantitation experiments, the mean fluorescent intensity (MFI) of a gated population was measured. Compensation to removed fluorescent spectral overlap was performed for each experimental measurement. Subtraction of either negative controls or cells treated with isotype antibodies was performed on signaling and receptor quantitation data respectively to remove background signal. Cells which were measured to display fluorescent intensities above $1,000,000$ were excluded from analysis during signaling experiments. Pearson correlation coefficients $\left(\mathrm{R}^{2}\right)$ values were used to describe model accuracy when predicting signaling response to IL-2 and IL-2 muteins. The $\mathrm{K}_{\mathrm{x}}{ }^{*}$ parameter was fit using least-squares fitting and the Broyden-Fletcher-Goldfarb-Shanno minimization algorithm as implemented in SciPy.

\section{References}

1. W. J. Leonard, J.-X. Lin, J. J. O’Shea, The yc Family of Cytokines: Basic Biology to Therapeutic Ramifications, Immunity 50, 832-850 (2019).

386 2. M. Noguchi, H. Yi, H. M. Rosenblatt, A. H. Filipovich, S. Adelstein, W. S. Modi, O. W. combined immunodeficiency in humans., Cell 73, 147-57 (1993). 
3. P. P. Zenatti, D. Ribeiro, W. Li, L. Zuurbier, M. C. Silva, M. Paganin, J. Tritapoe, J. A. Hixon, A. B. Silveira, B. A. Cardoso, L. M. Sarmento, N. Correia, M. L. Toribio, J. Kobarg, M. Horstmann, R. Pieters, S. R. Brandalise, A. A. Ferrando, J. P. Meijerink, S. K. Durum, J. A. Yunes, J. T. Barata, Oncogenic IL7R gain-of-function mutations in childhood T-cell acute lymphoblastic leukemia, Nature Genetics 43, 932-939 (2011). K. Weiskopf, H. Kohrt, J. E. Foley, S. Rajagopalan, E. O. Long, D. H. Fowler, T. A. Waldmann, K. C. Garcia, W. J. Leonard, Interleukin-2 activity can be fine-tuned with engineered receptor signaling clamps., Immunity 42, 826-38 (2015).

398 5. J. B. Spangler, J. Tomala, V. C. Luca, K. M. Jude, S. Dong, A. M. Ring, P. Votavova, M. Pepper, M. Kovar, K. C. Garcia, Antibodies to Interleukin-2 Elicit Selective T Cell Subset Potentiation through Distinct Conformational Mechanisms., Immunity 42, 815-25 (2015). 6. A. M. Farhat, A. C. Weiner, C. Posner, Z. S. Kim, B. Orcutt-Jahns, S. M. Carlson, A. S. Meyer, Modeling Cell-Specific Dynamics and Regulation of the Common Gamma Chain Cytokines, Cold Spring Harbor Laboratory (2020), doi:10.1101/778894. Campbell, M. A. Gavin, An IL-2 mutein engineered to promote expansion of regulatory T cells arrests ongoing autoimmunity in mice, Science Immunology 5, eaba5264 (2020). Waldhauer, A. Freimoser-Grundschober, E. Moessner, P. Umana, C. Klein, R. J. Hosse, L. S. Wicker, L. B. Peterson, Sustained in vivo signaling by long-lived IL-2 induces prolonged increases of regulatory T cells., J Autoimmun 56, 66-80 (2014).

411 9. C. M. Csizmar, J. R. Petersburg, T. J. Perry, L. Rozumalski, B. J. Hackel, C. R. Wagner, Multivalent Ligand Binding to Cell Membrane Antigens: Defining the Interplay of Affinity, Valency, and Expression Density., J Am Chem Soc 141, 251-261 (2018). Z. Wang, Diphtheria toxin-based bivalent human IL-2 fusion toxin with improved efficacy for targeting human CD25(+) cells., J Immunol Methods 405, 57-66 (2014).

417 11. L. L. Kiessling, J. E. Gestwicki, L. E. Strong, Synthetic Multivalent Ligands as Probes of Signal Transduction, Angewandte Chemie International Edition 45, 2348-2368 (2006).

419 12. I. Moraga, J. B. Spangler, J. L. Mendoza, M. Gakovic, T. S. Wehrman, P. Krutzik, K. C. Garcia, Synthekines are surrogate cytokine and growth factor agonists that compel signaling through non-natural receptor dimers, eLife 6, e22882 (2017).

422 13. S. Gillies, Y. Lan, B. Brunkhorst, W.-K. Wong, Y. Li, K.-M. Lo, Bi-functional cytokine 
14. S. J. York, L. S. Arneson, W. T. Gregory, N. M. Dahms, S. Kornfeld, The rate of internalization of the mannose 6-phosphate/insulin-like growth factor II receptor is enhanced by multivalent ligand binding., J Biol Chem 274, 1164-71 (1999).

428 15. R. Varma, K. Liu, K. Avery, R. Rashid, S. Schubbert, I. Leung, C. Bonzon, L. Bogaert, J. Desjarlais, M. J. Bernett, Regulatory T Cell Selective IL-2-Fc Fusion Proteins for the Treatment of Autoimmune Diseases, Blood 132, 3709-3709 (2018).

16. M. Murphy, S. D. Taylor, A. S. Meyer, Structured decomposition improves systems serology prediction and interpretation, Cold Spring Harbor Laboratory (2021), doi:10.1101/2021.01.03.425138.

17. Z. C. Tan, A. S. Meyer, A general model of multivalent binding with ligands of heterotypic subunits and multiple surface receptors, Cold Spring Harbor Laboratory (2021), doi:10.1101/2021.03.10.434776.

437 18. R. A. Robinett, N. Guan, A. Lux, M. Biburger, F. Nimmerjahn, A. S. Meyer, Dissecting 438 FcyR Regulation through a Multivalent Binding Model., Cell Syst 7, 41-48.e5 (2018).

19. A. S. Perelson, C. DeLisi, Receptor clustering on a cell surface. I. theory of receptor crosslinking by ligands bearing two chemically identical functional groups (Elsevier, 1980;

441 https://www.sciencedirect.com/science/article/abs/pii/0025556480900176).

442 20. J. D. Stone, J. R. Cochran, L. J. Stern, T-cell activation by soluble MHC oligomers can be 443 described by a two-parameter binding model., Biophys $J$ 81, 2547-57 (2001).

444 21. R. F. Pass, C. Hutto, R. Ricks, G. A. Cloud, Increased rate of cytomegalovirus infection 445 among parents of children attending day-care centers., N Engl J Med 314, 1414-8 (1986).

446 22. L. T. Yamamoto, H. N. Wigder, D. J. Fligner, M. Rauen, R. A. Dershewitz, Relationship of 447 bacteremia to antipyretic therapy in febrile children., Pediatr Emerg Care 3, 223-7 (1987).

448 23. Y. Mazor, K. F. Sachsenmeier, C. Yang, A. Hansen, J. Filderman, K. Mulgrew, H. Wu, W. F. Dall'Acqua, Enhanced tumor-targeting selectivity by modulating bispecific antibody binding affinity and format valence, Scientific Reports 7, 40098 (2017).

24. Z. C. Tan, B. Orcutt-Jahns, A. S. Meyer, A quantitative view of strategies to engineer cellselective ligand binding, Cold Spring Harbor Laboratory (2020), doi:10.1101/2020.11.25.398974.

456 (2017).

457 26. I. Moraga, J.B. Spangler, J.L. Mendoza, M. Gakovic, T.S. Wehrman, P. Krutzik, K.C. 458 Garcia, Synthekines are surrogate cytokine and growth factor agonists that compel signaling 459 through non-natural receptor dimers, eLife (2017).

460 27. Á. M. Cuesta, N. Sainz-Pastor, J. Bonet, B. Oliva, L. Álvarez-Vallina, Multivalent 461 antibodies: when design surpasses evolution, Trends in Biotechnology 28, 355-362 (2010). 
28. S. J. York, L. S. Arneson, W. T. Gregory, N. M. Dahms, S. Kornfeld, The Rate of Internalization of the Mannose 6-Phosphate/Insulin-like Growth Factor II Receptor Is Enhanced by Multivalent Ligand Binding, Journal of Biological Chemistry 274, 1164-1171 (1999).

30. L. B. Peterson, C. J. M. Bell, S. K. Howlett, M. L. Pekalski, K. Brady, H. Hinton, D. Sauter, J. A. Todd, P. Umana, O. Ast, I. Waldhauer, A. Freimoser-Grundschober, E. Moessner, C. Klein, R. J. Hosse, L. S. Wicker, A long-lived IL-2 mutein that selectively activates and expands regulatory T cells as a therapy for autoimmune disease, Journal of Autoimmunity 95, 1-14 472 (2018).

473 31. C. Ye, D. Brand, S. G. Zheng, Targeting IL-2: an unexpected effect in treating 474 immunological diseases, Signal Transduction and Targeted Therapy 3, 2 (2018).

475 32. J. P. Siegel, R. K. Puri, Interleukin-2 toxicity., Journal of Clinical Oncology 9, 694-704 476 (1991).

477 33. A. Carr, S. Emery, A. Lloyd, J. Hoy, R. Garsia, M. French, G. Stewart, G. Fyfe, D. A. Cooper, Australian IL-2 Study Group, Outpatient Continuous Intravenous Interleukin-2 or Subcutaneous, Polyethylene Glycol-Modified Interleukin-2 in Human Immunodeficiency VirusInfected Patients: A Randomized, Controlled, Multicenter Study, The Journal of Infectious Diseases 178, 992-999 (1998).

482 34. D.-A. Silva, S. Yu, U. Y. Ulge, J. B. Spangler, K. M. Jude, C. Labão-Almeida, L. R. Ali, A. Quijano-Rubio, M. Ruterbusch, I. Leung, T. Biary, S. J. Crowley, E. Marcos, C. D. Walkey, B. D. Weitzner, F. Pardo-Avila, J. Castellanos, L. Carter, L. Stewart, S. R. Riddell, M. Pepper, G. J. L. Bernardes, M. Dougan, K. C. Garcia, D. Baker, De novo design of potent and selective mimics of IL-2 and IL-15, Nature 565, 186-191 (2019). Cell-type-restricted anti-cytokine therapy: TNF inhibition from one pathogenic source, Proceedings of the National Academy of Sciences 113, 3006-3011 (2016).

493 37. J. B. Spangler, I. Moraga, J. L. Mendoza, K. C. Garcia, Insights into Cytokine-Receptor 494 Interactions from Cytokine Engineering, Annual Review of Immunology 33, 139-167 (2015).

495 38. J. S. Peraino, H. Zhang, P. V. Rajasekera, M. Wei, J. C. Madsen, D. H. Sachs, C. A. Huang, 

Wang, Ontak-like human IL-2 fusion toxin, Journal of Immunological Methods 448, 51-58

500 (2017).

501 40. Z. Qi, Y. Qiu, Z. Wang, H. Zhang, L. Lu, Y. Liu, D. Mathes, E. A. Pomfret, D. Gao, S. Lu, 502 Z. Wang, A novel diphtheria toxin-based bivalent human EGF fusion toxin for treatment of head 503 and neck squamous cell carcinoma, Molecular Oncology 15, 1054-1068 (2021).

504 41. J. Kossaifi, Y. Panagakis, A. Anandkumar, M. Pantic, TensorLy: Tensor Learning in Python 505 (arXiv, 2018; https://arxiv.org/abs/1610.09555).

506 42. A. M. Farhat, A. C. Weiner, C. Posner, Z. S. Kim, B. Orcutt-Jahns, S. M. Carlson, A. S.

507 Meyer, Modeling cell-specific dynamics and regulation of the common gamma chain cytokines, 508 Cell Reports 35, 109044 (2021).

509 43. T. Ishino, M. Wang, L. Mosyak, A. Tam, W. Duan, K. Svenson, A. Joyce, D. M. O’Hara, L. 510 Lin, W. S. Somers, R. Kriz, Engineering a Monomeric Fc Domain Modality by N-Glycosylation 511 for the Half-life Extension of Biotherapeutics, Journal of Biological Chemistry 288, 16529-

51216537 (2013).

\section{Acknowledgements:}

514 Funding: This work was supported by a research agreement with Visterra, Inc.

515 Author contributions statement: A.S.M. and S.M.C. conceived of the study. C.P. and S.M.C. 516 performed the PBMC experiments and engineered the IL-2 fusion proteins. A.S.M, B.O.J., 517 E.M.S., and P.C.E. performed the computational analysis. All authors helped to design 518 experiments and/or analyze the data. All authors contributed to writing the paper.

519 Competing interests: S.M.C. and C.P. are employees of Visterra Inc. A.S.M. has filed an 520 invention disclosure on the use of multivalent cytokines to enhance cell type selective responses.

521 Data Sharing Plan: All analysis was implemented in Python v3.9 and can be found at $522 \mathrm{https}$ //github.com/meyer-lab/gc-valent, release 1.0, along with all experimental data. 


\section{$523 \quad$ Figures}
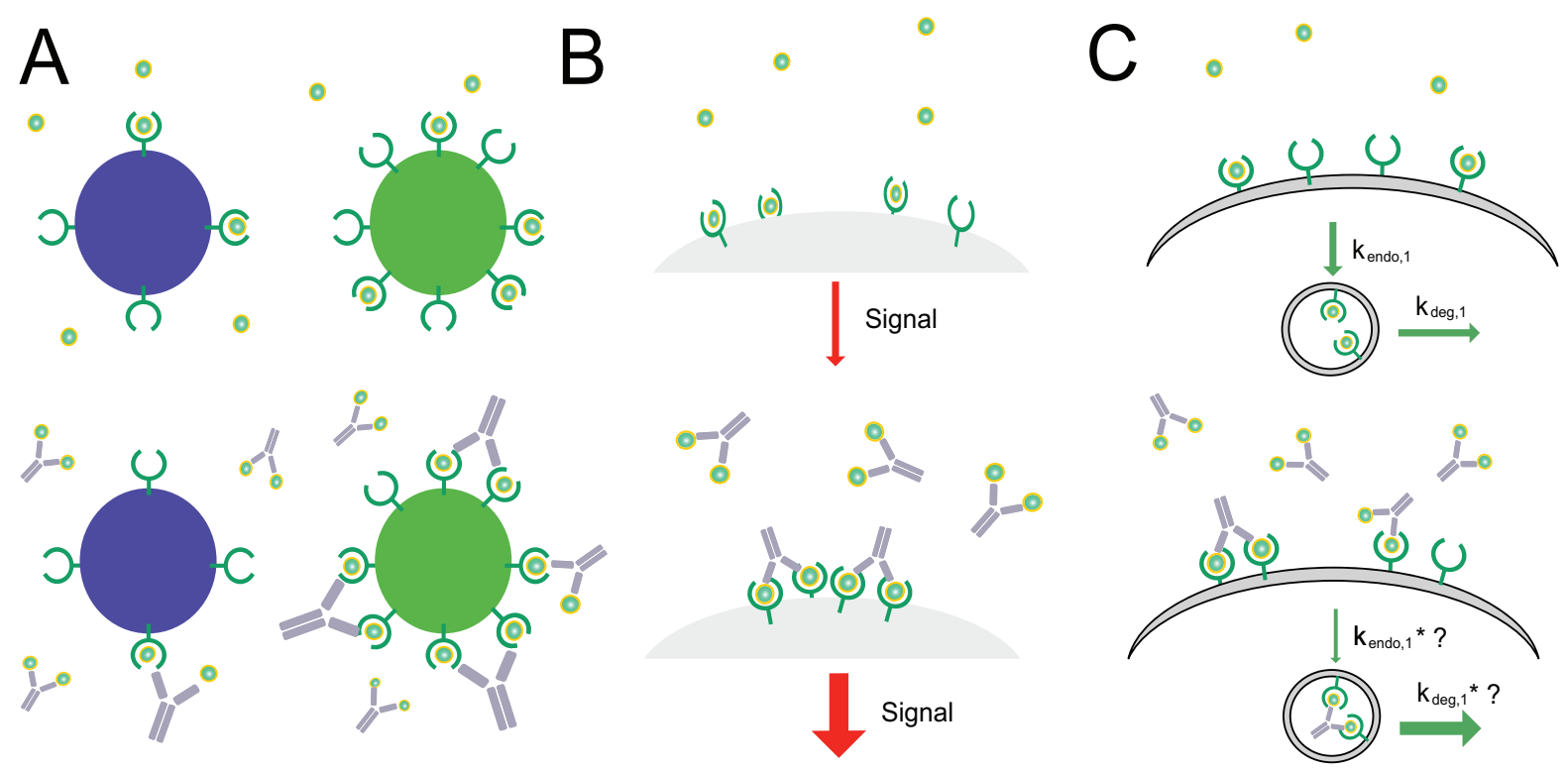

525 Fig. 1. Possible effects of bivalent Fc-cytokine formats. (A) Multivalent ligands are known to bind to surface receptors with altered functional affinities that vary as a function of the number of receptors displayed by the cell. (B) Cartoon illustrating how ligand valency may alter signal transduction dynamics. Multivalent binding has been shown to be critical in some signaling pathways via the local aggregation of active signaling complexes. (C) Multivalently bound receptors have been shown to be degraded intracellularly at a rate differing from monovalently bound receptors and have also been shown to possess altered in vivo pharmacokinetics. 
a
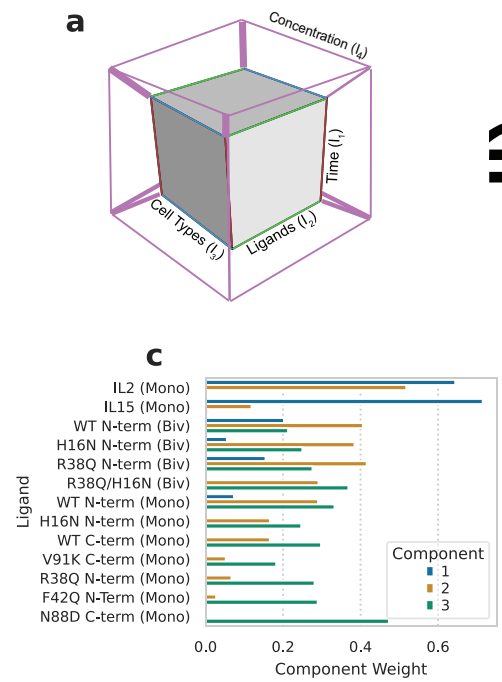

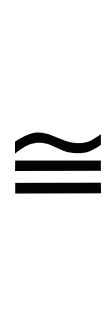

d

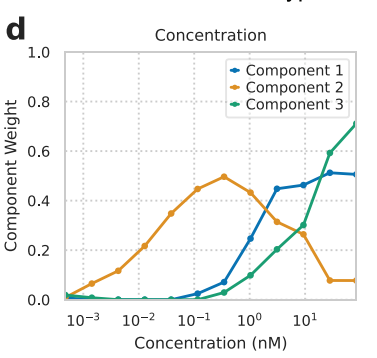

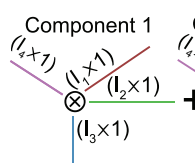
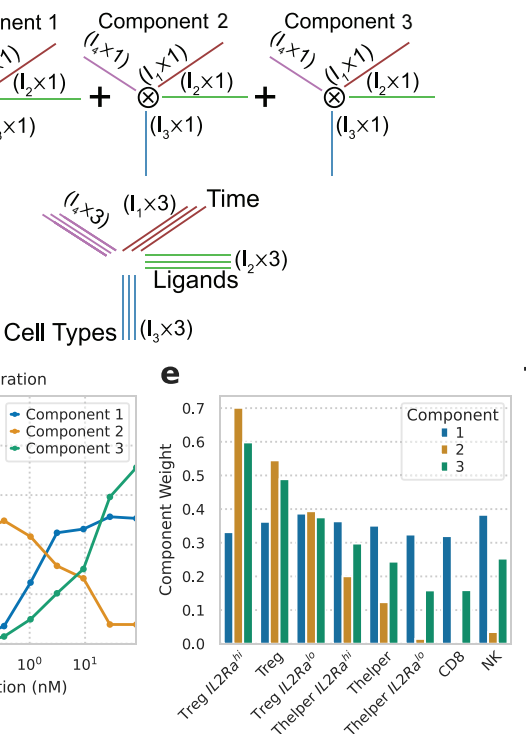

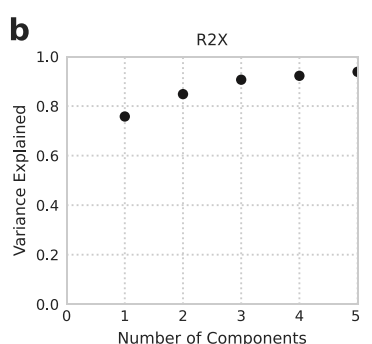

f

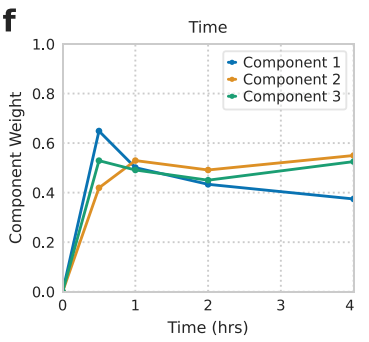

533 Fig. 2. pSTAT5 response varies in a cell type- and treatment-specific manner. (A) Schematic representation of non-negative canonical polyadic (CP) decomposition. Experimental pSTAT5 measurements are arranged in a tensor according to the duration of treatment, ligand used, cytokine concentration, and cell type. CP decomposition then helps to visualize this space. (B) Percent variance reconstructed (R2X) versus the number of components used. (C) Component values for each IL-2 form. (D) Component values representing the effect of IL-2 concentration. (E) Component values representing cell type specificity. (F) Component values for the effect of treatment duration. 
a
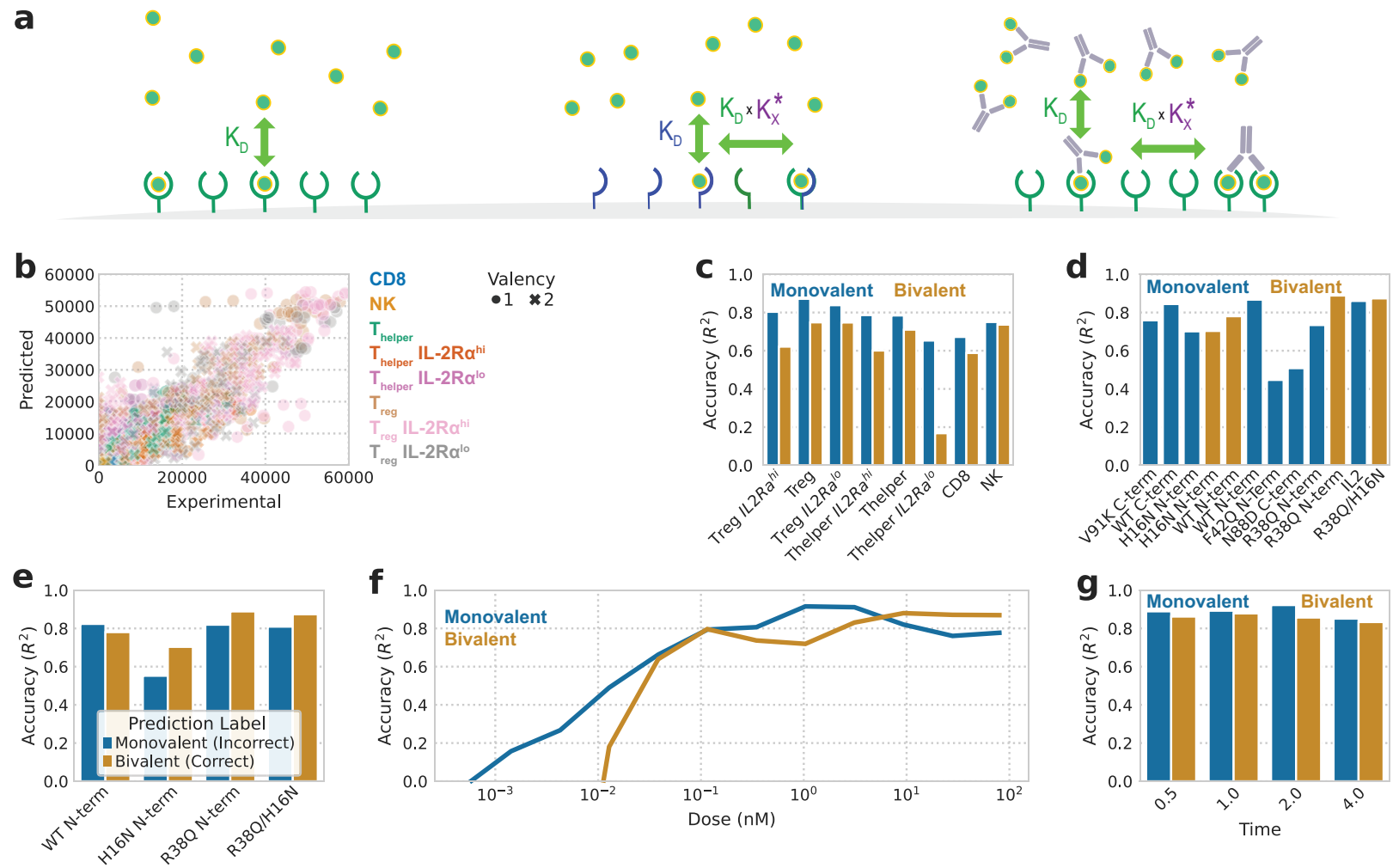

Fig. 3. Responses are predicted by a simple multivalent binding model. All accuracies are calculated as a Pearson's correlation $\mathrm{R}^{2}$ score for experimental cytokine responses at the 30 minute and one hour time points. (A) A simplified cartoon of the model. Initial association of multivalent ligands proceeds according to monovalent affinity, and subsequent binding events proceed with that affinity scaled by the $\mathrm{K}_{\mathrm{x}}^{*}$ parameter. (B) Experimentally measured vs. predicted pSTAT5 responses for all cell types, ligands, and concentrations. Each point represents a single experimental measurement (N=1). (C,D) Model's accuracy subset by cell type $(\mathbf{C})$ and ligand (D) for all mono- and bivalent IL-2 muteins. (E) Model's accuracy when predicting the responses of all cell types to bivalent ligands either correctly as dimers, or as IL-2 monomers. $(\mathbf{F}, \mathbf{G})$ Model's accuracy subset by concentration (F) for all ligands and time (G) for all ligands, concentrations, and cell types. 

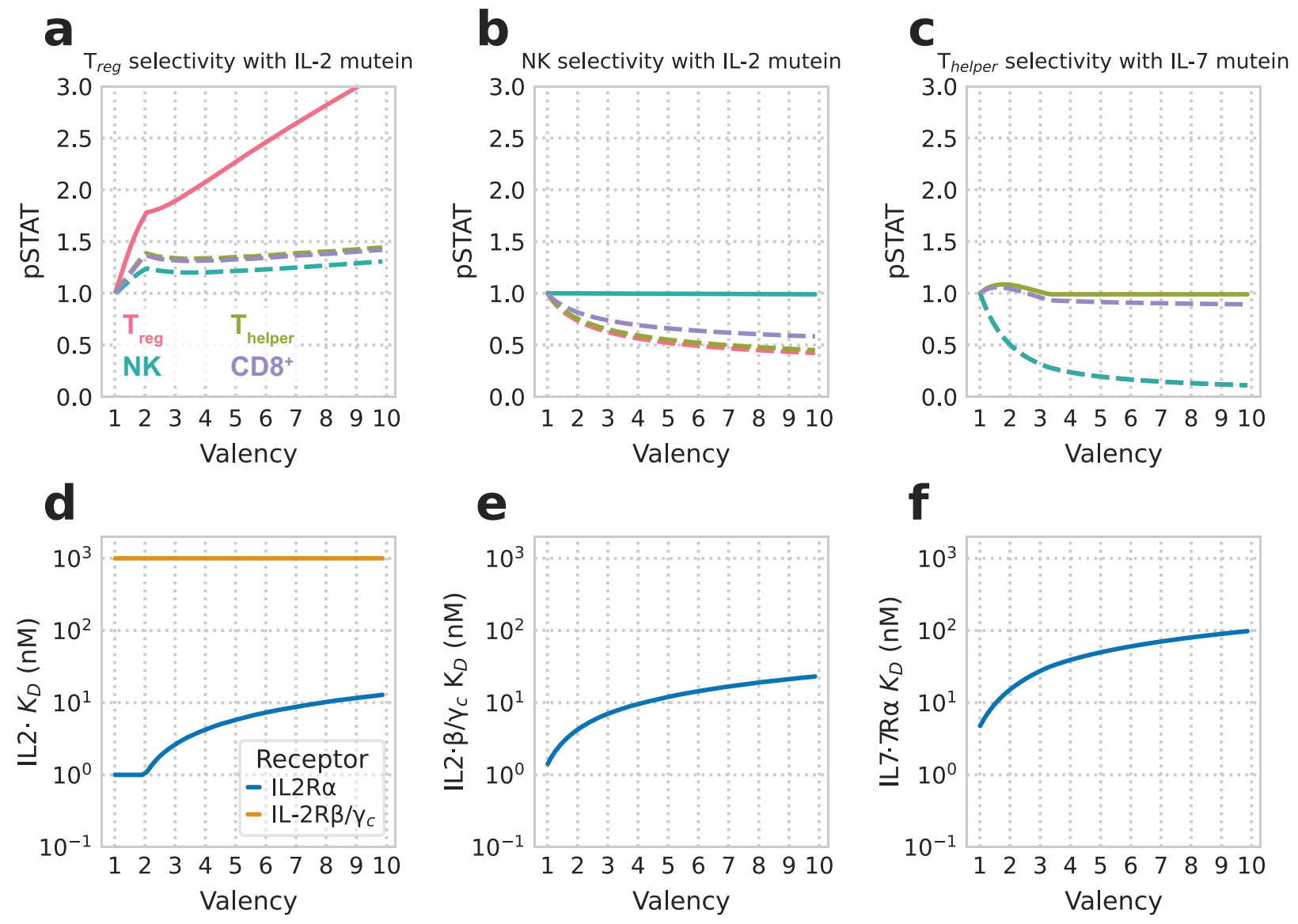

Fig. 4. Multivalency with coordinate affinity adjustments can enhance selectivity. Affinities

555 were allowed to vary across $\mathrm{K}_{\mathrm{DS}}$ of $10 \mathrm{pM}-1 \mu \mathrm{M}$ while $K_{x}^{*}$ was fixed at its fitting optimum. All optimizations were performed using a concentration of $1 \mathrm{nM}$. Selectivity was calculated as the ratio of predicted pSTAT5 in target cells to the mean pSTAT5 predicted in off-target cells. (A,C,E) Signaling response of $\mathrm{T}_{\text {reg }}, \mathrm{NK}$ cells, and $\mathrm{T}_{\text {helper }}$ cells predicted for ligand of optimal selectivity at different valencies. Response predictions were normalized to each population's response for the monovalent case. Selectivity for $\mathrm{T}_{\text {reg }} \mathrm{S}$ and NK cells were derived from IL-2 muteins, and selectivity for $T_{\text {helper }} \mathrm{S}$ was calculated using IL-7 muteins. (B,D,F) Optimal receptorligand dissociation constants for each ligand optimized for selectivity. Mutein affinity for IL-2R $\alpha$ for IL-7 muteins. 